\title{
Quality of life impairment due to chronic rhinosinusitis in asthmatics is mediated by asthma control*
}

\author{
Katie M. Phillips ${ }^{1,2}$, Rehab Talat ${ }^{3}$, David S. Caradonna ${ }^{1,4}$, Stacey T. Gray ${ }^{1,2}$, \\ Ahmad R. Sedaghat ${ }^{3}$ \\ 'Department of Otolaryngology, Harvard Medical School, Boston, MA, USA \\ 2Department of Otolaryngology, Massachusetts Eye and Ear Infirmary, Boston, MA, USA \\ ${ }^{3}$ Department of Otolaryngology - Head \& Neck Surgery, University of Cincinnati College of Medicine, Cincinnati, Ohio, USA, \\ ${ }^{4}$ Division of Otolaryngology, Beth Israel Deaconess Medical Center, Boston, MA, USA
}

Rhinology 57: 6, $430-435,2019$

https://doi.org/10.4193/Rhin19.207

*Received for publication:

May 30, 2019

Accepted: July 15, 2019

\begin{abstract}
Background: Chronic rhinosinusitis (CRS) and asthma, when comorbid, may influence each other's disease course and decrease quality of life (QOL). Our objective was to determine if poorer asthma control due to CRS symptoms could be a mechanism for decreased QOL in asthmatic CRS patients.
\end{abstract}

Methods: A total of 120 asthmatic CRS patients were recruited. CRS symptom burden was measured using the 22 -item Sinonasal Outcome Test (SNOT-22) and patient-reported CRS symptom control, general health-related QOL was measured using the visual analog scale of the 5-dimensional EuroQol quality of life survey (EQ-5D VAS), and asthma control was measured using the Asthma Control Test (ACT). Association was sought between these outcome measures. A mediation model was created and validated to show that asthma control mediated the association between CRS symptom burden and decreased general health-related QOL.

Results: ACT score was associated with SNOT-22, EQ-5D VAS was associated with SNOT-22 score, and EQ-5D VAS was associated with ACT score. A statistically significant mediation effect for ACT score in the association between SNOT-22 and EQ-5D VAS), which represented $22.1 \%$ of the total effect of SNOT-22 on EQ-5D VAS, was identified. Similar findings were made for patientreported CRS symptom control instead of SNOT-22 score.

Conclusions: In asthmatic CRS patients, a sizeable portion of CRS impact on QOL is indirectly mediated through the effect of CRS on poorer asthma control which may then drive decreased QOL.

Key words: chronic rhinosinusitis, asthma, general health-related quality of life, asthma control, mediation

\section{Introduction}

Chronic rhinosinusitis (CRS) is an inflammatory disorder of the sinonasal mucosa that affects approximately $10 \%$ of the population, causes a significant quality of life (QOL) detriment and leads to billions of dollars of costs to society every year ${ }^{(1)}$. The impact of CRS exacerbations and CRS-associated sinonasal symptomatology on reduced QOL has been well-described ${ }^{(2-4)}$. Asthma is a common comorbidity in CRS patients and it may be a significant source of morbidity in these patients ${ }^{(5)}$. Epidemiologic and clinical associations as well as common pathophysiologic mechanisms between asthma and CRS suggest the possibility of exacerbation of one disease due to the other ${ }^{(5)}$. Greater
CRS symptoms and more frequent acute exacerbations of CRS (AECRS) have been shown to be associated with worse asthma control ${ }^{(6-8)}$. Although it is likely that each disease may exacerbate the other, the preponderance of data suggests a dominant role for CRS in the exacerbation of asthma ${ }^{(9,10)}$.

Asthma is associated with significant decreases in QOL which are compounded by the morbidity of asthma exacerbations. As a chronic disease of the lower airways, characterized by obstruction and hyperreactivity of the lower airways, that manifests with symptoms such as wheezing, cough, and shortness of breath, asthma can be physically, emotionally and socially debilitating ${ }^{(11,12)}$. Acute exacerbations of asthma also take a similar toll 
on patients and are associated with significant disability, morbidity and even mortality ${ }^{(13,14)}$. Prior studies have suggested the possibility of CRS and poor control of its symptoms to negatively impact comorbid asthma ${ }^{(6-8)}$. The ability of CRS to exacerbate asthma severity and worsen asthma control may therefore serve as one mechanism through which CRS decreases QOL. Because decreased general health-related $\mathrm{QOL}$ is the predominant consequence of CRS that translates to billions of dollars in direct and indirect costs-from patients seeking medical care and missing work, respectively_annually, we sought to determine if the impact of CRS on asthma control could serve as a distinct mechanism for decreased general health-related QOL in asthmatic CRS patients. It is known that CRS symptom burden and poor CRS symptom control are associated with decreased general health-related QOL ${ }^{(2,15)}$. We hypothesized that decreased asthma control would mediate these associations between CRS symptoms and decreased general health-related QOL. Such a mediation effect would demonstrate a mechanism for decreased QOL that is independent of the direct effects of sinonasal symptoms in asthmatic CRS patients and would provide greater evidence for the interactions between the upper and lower airways.

\section{Materials and methods}

\section{Study participants and design}

This cross-sectional cohort study was approved by our institution's Human Studies Committee. Patients, aged 18 years or older, meeting consensus guideline criteria for CRS ${ }^{(16)}$ as well as criteria for asthma ${ }^{(12)}$ were prospectively recruited over a one year period. All study participants provided informed consent. Exclusion criteria included comorbid diagnoses of vasculitis, cystic fibrosis, sarcoidosis, and immunodeficiency as well as endoscopic sinus surgery within the past 6 months (to remove the confounding effects of recent sinus surgery).

All data were collected at the time of enrollment. The age, gender, race, history of previous sinus surgery, intranasal corticosteroid use (as spray or irrigation), inhaled corticosteroid use and smoking history of all participants were recorded. Any patient with a past history of smoking was considered a smoker for this study ${ }^{(17,18)}$. Aeroallergen hypersensitivity was determined based on a history of positive skin or serological testing. Participants were assessed by the evaluating rhinologist for nasal polyps using endoscopy and then asked to rate control of their CRS symptoms over the last 6 weeks as "Not at all", "A little", "Somewhat", "Very" or "Completely", as previously described ${ }^{(15,19)}$, where "Very" and "Completely" controlled symptoms were considered well-controlled and all other levels of symptom control were considered poorly-controlled CRS symptoms. Finally, participants were given three standardized surveys, the 22-item Sinonasal Outcome Test (SNOT-22) ${ }^{(20)}$ to measure symptom severity, the 5-dimensional EuroQol quality of life survey (EQ-5D) from which the visual analog scale (EQ-5D VAS) was used as a measure of general health-related $\mathrm{QOL}^{(21,22)}$, and the Asthma Control Test (ACT) ${ }^{(23)}$ to assess asthma control.

\section{Statistical analysis}

All analysis was performed using the statistical software package $R$ (www.r-project.org). Univariate and multivariable associations with ACT score or EQ-5D VAS as dependent variables were calculated using linear regression. Multivariable linear regression models controlled for participant age, gender, smoking history, presence of nasal polyps, aeroallergen hypersensitivity, intranasal corticosteroid use and corticosteroid inhaler use. The mediation effect of asthma control (reflected by ACT score) in the association between SNOT-22 score or control of CRS symptoms (as independent variables) and EQ-5D VAS score (as dependent variable) were determined using Sobel's indirect test for mediation and bootstrapping over our dataset with 1000 iterations. When a significant mediation effect was found, we confirmed that all arms of the mediation model consisted of statistically significant associations.

\section{Results}

\section{Study participants}

The demographic and clinical characteristics of our 120 study participants are summarized in Table 1. There was a female predominance (62.5\%) compared to males (37.5\%). As expected, a high fraction of patients tested positive for aeroallergen hypersensitivity (68.3\%) and had nasal polyps (70.0\%). The mean SNOT-22 score was 41.5 (SD: 24.1 ) and only $38.3 \%$ rated their symptoms to be well-controlled. The mean ACT score was 20.0 (SD: 5.4 ) with $34.2 \%$ of patients have an ACT score less than 20 , indicating poor asthma control. The mean EQ-5D VAS was 67.4 (SD: 21.5).

Asthma control partially mediates the relationship between CRS symptom burden and general health-related QOL It is well described that the burden of CRS symptoms is associated with decreased general health-related $\mathrm{QOL}{ }^{(1,4)}$. However, we sought to determine if the level of asthma control mediates this association in asthmatic CRS patients. We first checked the relationships between SNOT-22 score, ACT score and EQ-5D VAS. Associations between CRS symptom burden (SNOT-22), asthma control (ACT score) and general health-related QOL (EQ-5D VAS) are shown in Figure 1. In this cohort, ACT score (as dependent variable) was associated with SNOT-22 score on univariate (linear regression coefficient $[\beta]=-0.09,95 \% \mathrm{Cl}:-0.13$ to $-0.05, \mathrm{p}<0.001$ ) and multivariable linear regression ( $\beta=-0.09,95 \% \mathrm{Cl}:-0.12$ to $-0.05, \mathrm{p}<0.001$ ). EQ-5D VAS (as dependent variable) was also associated with SNOT-22 score on univariate $(\beta=-0.36,95 \% \mathrm{Cl}$ : -0.51 to $-0.21, p<0.001)$ and multivariable linear regression ( $\beta$ $=-0.33,95 \% \mathrm{Cl}:-0.49$ to $-0.19, \mathrm{p}<0.001$ ). EQ-5D VAS (as dependent 
Table 1. Characteristics of study participants.

\begin{tabular}{|lc|}
\hline & $\begin{array}{c}\text { Study participants } \\
(\mathbf{n}=120)\end{array}$ \\
\hline Demographics & \\
\hline Age, mean in years, (SD) & $50.2(17.0)$ \\
\hline $\begin{array}{l}\text { Gender } \\
\text { Male }\end{array}$ & $37.5 \%$ \\
$\quad$ Female & $62.5 \%$ \\
\hline Smoking & $35.8 \%$ \\
\hline Aeroallergen hypersensitivity & $68.3 \%$ \\
\hline CRS and asthma characteristics & \\
\hline Nasal polyps & $70.0 \%$ \\
\hline Previous sinus surgery & $50.0 \%$ \\
\hline Intranasal steroid use & $62.5 \%$ \\
\hline Steroid inhaler use & $50.0 \%$ \\
\hline SNOT-22 score, mean (SD) & $41.5(24.1)$ \\
\hline Patient-reported CRS symptom control & \\
\hline Poorly controlled & $61.7 \%$ \\
\hline Well-controlled & $38.3 \%$ \\
\hline EQ-5D VAS, mean (SD) & $67.4(21.5)$ \\
\hline ACT score, mean (SD) & $20.0(5.4)$ \\
\hline
\end{tabular}

variable) was found to similarly be associated with ACT score on univariate $(\beta=1.40,95 \% \mathrm{Cl}: 0.72$ to $2.08, \mathrm{p}<0.001)$ and multivariable linear regression ( $\beta=1.07,95 \% \mathrm{Cl}$ : 0.36 to $1.78, p=0.004)$. Given these significant associations, we assumed a model whereby CRS symptoms, as the independent variable, drive decreases in QOL and assessed whether the level of asthma control (at least partially) mediated this relationship. In other words, was a portion of the association between CRS symptom burden and decreased QOL due to an effect that CRS symptoms may have on asthma control that then leads to decreased QOL? We found a statistically significant mediation effect for ACT score in the association between SNOT-22 and EQ-5D VAS (mediation effect $=-0.08,95 \% \mathrm{Cl}$ : -0.15 to $-0.02, p=0.012$ ), which represented $22.1 \%$ of the total effect of SNOT-22 on EQ-5D VAS.

Asthma control partially mediates the relationship between CRS symptom control and general health-related QOL Because CRS symptom burden, although measured on a continuum with the SNOT-22, may not necessarily translate linearly to general QOL burden ${ }^{(24)}$, we also assessed whether the relationship between patient-reported CRS symptom control (as a complementary measure to the SNOT-22) and general health-related QOL was mediated by asthma control. We again first checked the relationships between patient-reported control level, ACT score and EQ-5D VAS. We found statistically significant associations between well-controlled vs. poorly controlled CRS symptoms, ACT score and EQ-5D VAS (Figure 2). Specifically, well-controlled CRS symptoms were significantly

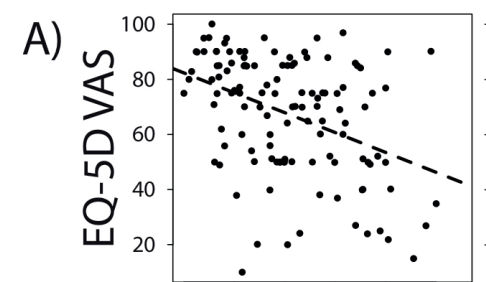

B)
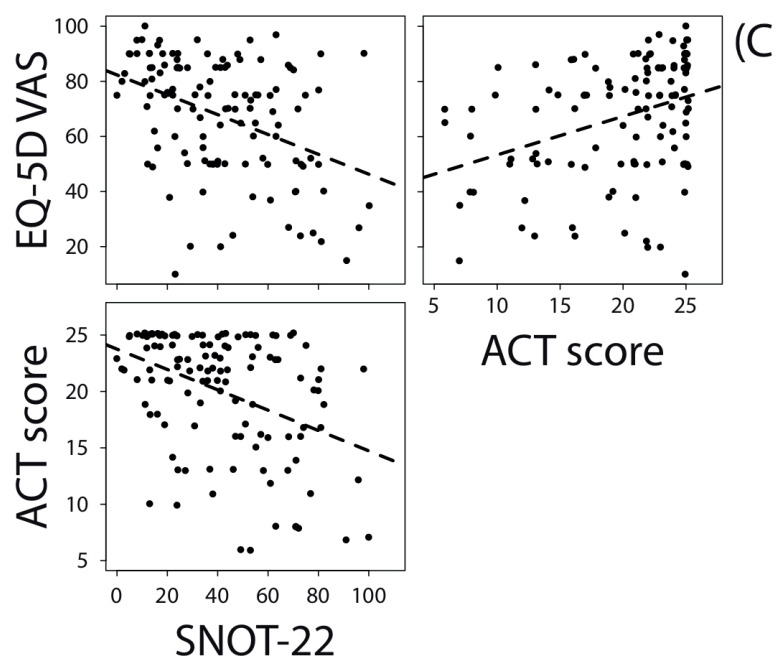

ACT score

Figure 1. Scatterplots of (A) EQ-5D VAS vs. SNOT-22, (B) ACT score vs. SNOT-22, and (C) EQ-5D VAS vs. ACT score. The dashed lines of best fit are superimposed.

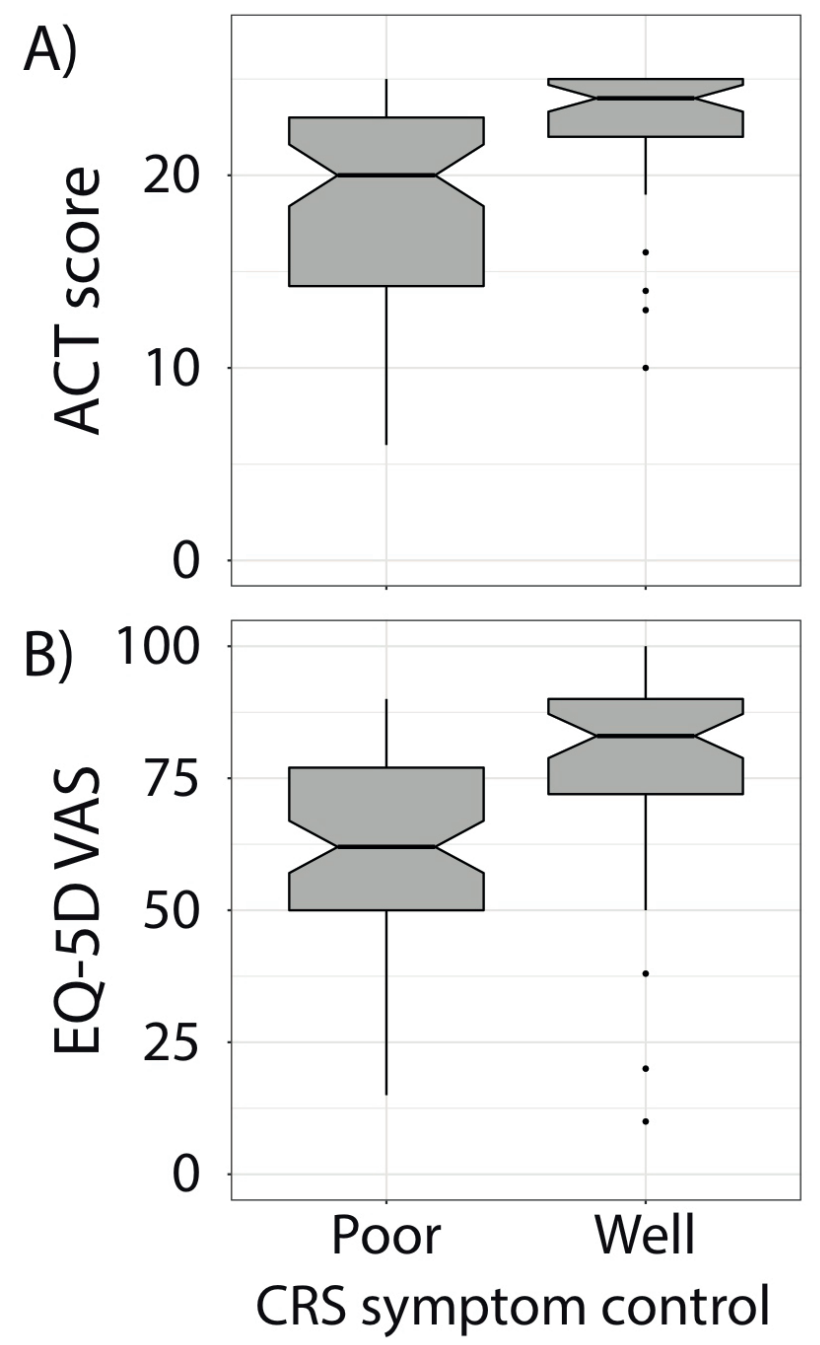

Figure 2. Bar plots of (A) ACT score and (B) EQ-5D VAS stratified according to patient-reported CRS symptom control. 
associated with higher ACT score on univariate $(\beta=1.61,95 \% \mathrm{Cl}$ : $0.82-2.41, p<0.001)$ and multivariable $(\beta=1.55,95 \% \mathrm{Cl}: 0.65-$ 2.44, $p=0.001$ ) linear regression and also associated with higher EQ-5D VAS on univariate $(\beta=4.18,95 \% \mathrm{Cl}: 2.34-6.02, \mathrm{p}=0.001)$ and multivariable ( $\beta=3.84,95 \% \mathrm{Cl}: 1.83-5.84, p<0.001)$ linear regression. These results also suggested the possibility of asthma control mediating the relationship between CRS symptom control and decreased QOL in these asthmatic CRS patients. When we investigated this possible mediation relationship, we found a statistically significant mediation effect for ACT score between patient-reported CRS symptom control and EQ-5D VAS (mediation effect $=4.08,95 \% \mathrm{Cl}: 1.13$ to $7.52, \mathrm{p}=0.006$ ), which represented $24.9 \%$ of the total effect of patient-reported CRS symptom control on EQ-5D VAS.

\section{Discussion}

The predominant impact that CRS has on patients is a significant QOL detriment-comparable to that caused by other severe chronic conditions such as heart disease or diabetes-that is due to chronic sinonasal symptomatology ${ }^{(3,4)}$. Further investigation to determine the mechanisms driving this decrease in QOL is paramount to identifying treatment targets for improving QOL and ultimately decreasing CRS-related societal costs. We have previously surmised and shown that decreased QOL may be driven by independent manifestations of the disease, such as chronic baseline symptomatology and the frequency of AECRS ${ }^{(3,6)}$. Exacerbation of pulmonary disease may be another distinct disease manifestation that decreases QOL in affected patients. CRS is epidemiologically associated with asthma, with these two diseases commonly impacting each other's natural history of disease ${ }^{(5,7,8)}$. CRS in particular has been described as a driver of poor asthma outcomes. The severity of CRS symptomatology is associated with the degree of asthma control in asthmatic CRS patients, independent of patient characteristics or treatment with intranasal or inhaled corticosteroids ${ }^{(8)}$. The presence of CRS has also been associated with worse pulmonary function testing results in asthmatics ${ }^{(9)}$ and increased frequency of asthma exacerbations with increased frequency of asthma-related emergency room visits, hospitalizations, and systemic corticosteroid use ${ }^{(25)}$. In this study, we sought to determine whether the wellestablished relationship between CRS symptom burden and decreased QOL could be in part due to (i.e. mediated by) the impact of CRS disease burden on the lower airways. We focused on asthmatic CRS patients and found that asthma control did mediate the relationship between CRS disease burden-symptom burden and symptom control—and decreased general health-related QOL. In fact, the effect of CRS in decreasing QOL through its impact on asthma control was comparable to (20 $25 \%$ of) its direct effect on decreased QOL.

Past studies have demonstrated the direct impact of the CRS disease course to negatively impact comorbid asthma disease course. We have previously shown that the CRS symptom burden and AECRS frequency are negatively associated with poor asthma control, asthma exacerbation frequency and productivity loss in asthmatic CRS patients ${ }^{(6-8,26,27)}$. Other studies have also shown potentially direct evidence for the downstream exacerbation of pulmonary status by worsening CRS. For example, AECRS have been found to frequently precede asthma exacerbations and asthma-related ED visits and hospitalizations ${ }^{(10,28,29)}$.

While previous studies have reported a general association between CRS and worse pulmonary status in asthmatics ${ }^{(9,25,28)}$ our study provides further evidence for the continuum model for an interdependent relationship of CRS and asthma whereby the real-time status of one may impact or determine the status of the other (28). Our study also highlights that worsening pulmonary status may be a distinct mechanism through which CRS may cause a significant decrease in general health-related QOL in asthmatic CRS patients, and therefore a potential target for improving QOL in asthmatic CRS patients. This is also supported by previous work showing that treatment of CRS may improve asthma outcomes in asthmatic CRS patients ${ }^{(30,31)}$. Our study provides a framework for understanding how treatment of CRS may impact asthma control and general health-related QOL in asthmatic CRS patients. Our results establish the relative magnitudes of associations between CRS severity, asthma control and the QOL detriment further unveiling the complex interplay between these diseases. These results also identify asthma control as a potentially modifiable factor that can be addressed to improve general health-related QOL in asthmatic CRS patients.

Our results should be interpreted in the context of our study limitations. This was a cross-sectional study which showed association but no causality or responsiveness between changes in CRS symptom burden and asthma control. As a complement to CRS symptom burden measured by the SNOT-22, we also assessed patient-reported CRS symptom control by simply asking participants to rate how controlled their symptoms were. It is important recognize that this is not a measure of CRS disease control, a far more complex construct that may include not just symptom burden but other metrics of disease burden, such as acute CRS exacerbation frequency or the need for systemic medications ${ }^{(1,32)}$. Our study also did not factor in objective measures of CRS or asthma severity. Previous studies have shown poor correlation cross-sectionally between CRS symptom burden using SNOT-22 and objective CRS burden using nasal endoscopy ${ }^{(33)}$. However, there may be an association between objective CRS burden and asthma since asthma has been associated with worse radiographic CRS burden ${ }^{(33)}$. Nevertheless, we feel that the lack of objective metrics of disease does not reduce the validity of these results but instead is an exciting avenue for future work that is motivated by these results. Additionally, 
our study is performed in a general population of CRS patients and it is possible that the magnitude of mediation effects may vary in more distinct subsets of CRS patients based on clinical or molecular markers. That our associations were robust to controlling for clinical confounders such as the presence of polyps or aeroallergen hypersensitivity suggests that the presence of this mediation effect - that a portion of the impact of CRS on decreased QOL is through effects on the lower airway in asthmatics - will likely be present in most if not all clinically-defined subsets of CRS patients.

\section{Acknowledgements}

No funding was used to support this study.

The authors gratefully acknowledge the contribution of the patients who participated in this study.

\section{Authorship contribution}

KMP, RT, DSC, STG performed the study and wrote/revised the manuscript. ARS conceived, designed, and performed the study and wrote/revised the manuscript.

\section{Conflict of interest}

The authors declare no conflicts of interest.

\section{References}

1. Fokkens WJ, Lund VJ, Mullol Jet al. European Position Paper on Rhinosinusitis and Nasal Polyps 2012. RhinologySupplement 2012 (23):298.

2. Hoehle LP, Phillips KM, Bergmark RW Caradonna DS, Gray ST, Sedaghat AR. Symptoms of chronic rhinosinusitis differentially impact general health-related quality of life. Rhinology 2016; 54:316-322.

3. Phillips KM, Hoehle LP, Bergmark RW, Caradonna DS, Gray ST, Sedaghat AR. Acute Exacerbations Mediate Quality of Life Impairment in Chronic Rhinosinusitis. Allergy Clin Immunol Pract 2017; 5:422-426.

4. Speth MM, Hoehle LP, Phillips KM, Caradonna DS, Gray ST, Sedaghat AR. Changes in chronic rhinosinusitis symptoms differentially associate with improvement in general health-related quality of life. Ann Allergy Asthma Immunol 2018.

5. Rosati MG, Peters AT. Relationships among allergic rhinitis, asthma, and chronic rhinosinusitis. Am J Rhinol Allergy 2016; 30:44-47.

6. Banoub RG, Phillips KM, Hoehle LP, Caradonna DS, Gray ST, Sedaghat AR Relationship between chronic rhinosinusitis exacerbation frequency and asthma control. Laryngoscope 2018; 128:1033-1038.

7. Phillips KM, Hoehle LP, Bergmark RWet al. Chronic rhinosinusitis severity is associated with need for asthma-related systemic corticosteroids. Rhinology 2017; 55:211-217.

8. Phillips KM, Hoehle LP, Caradonna DS, Gray ST, Sedaghat AR. Association of severity of chronic rhinosinusitis with degree of comorbid asthma control. Ann Allergy Asthma Immunol 2016; 117:651-654.

9. Ek A, Middelveld RJ, Bertilsson Het al. Chronic rhinosinusitis in asthma is a negative predictor of quality of life: results from the Swedish GA(2)LEN survey. Allergy 2013 68:1314-1321.

10. Ikeda K, Yokoi H, Kusunoki Tet al. Bacteriology of recurrent exacerbation of postoperative course in chronic rhinosinusitis in relation to asthma. Auris, Nasus, Larynx $2011 ; 38: 469-473$

11. Reddel HK, Bateman ED, Becker Aet al. A summary of the new GINA strategy: a roadmap to asthma control. Eur Resp J 2015 46:622-639.

12. EPR-3. NAEPP expert panel report 3: guidelines for the diagnosis and treatment of asthma. Bethesda (MD): US Department of Health and Human Services; National Institutes of Health; National Heart, Lung, and Blood Institute, 2007.

13. Fuhlbrigge A, Peden D, Apter AJet al. Asthma outcomes: exacerbations. J Allergy Clin Immunol 2012; 129:S34-48.

14. Abrams EM, Becker AB, Szefler SJ. Where does worsening asthma end and an asthma exacerbation begin? Ann Allergy Asthma Immunol 2018

15. Gray ST, Hoehle LP, Phillips KM, Caradonna DS, Sedaghat AR. Patient-reported control of chronic rhinosinusitis symptoms is positively associated with general healthrelated quality of life. Clin Otolaryngol 2017; 42:1161-1166.

16. Rosenfeld RM, Piccirillo JF, Chandrasekhar SS et al. Clinical practice guideline (update): adult sinusitis. Otolaryngol Head Neck Surg. 2015: 152:S39.

17. Hoehle LP, Phillips KM, Caradonna DS, Gray ST, Sedaghat AR. A contemporary analysis of clinical and demographic factors of chronic rhinosinusitis patients and their association with disease severity. Ir J Med Sci 2018; 187:215-221

18. Phillips KM, Hoehle L, Bergmark RW, Caradonna DS, Gray ST, Sedaghat AR. Reversal of Smoking Effects on Chronic Rhinosinusitis after Smoking Cessation. Otolaryngol Head Neck Surg 2017; 157:737742

19. Gray ST, Phillips KM, Hoehle LP, Caradonna DS, Sedaghat AR. The 22-item Sino-Nasal Outcome Test accurately reflects patientreported control of chronic rhinosinusitis symptomatology. Int Forum Allergy Rhinol 2017; 7:945-951

20. Hopkins C, Gillett S, Slack R, Lund VJ, Browne JP. Psychometric validity of the 22-item Sinonasal Outcome Test. Clin Otolaryngol2009; 34:447-454.

21. EuroQol G. EuroQol--a new facility for the measurement of health-related quality of life. Health policy (Amsterdam, Netherlands) 1990; 16:199-208.

22. Hoehle LP, Phillips KM, Speth MM Caradonna DS, Gray ST, Sedaghat AR. Responsiveness and minimal clinically important difference for the EQ-5D in chronic rhinosinusitis. Rhinology 2019; 57:110-116.

23. Schatz M, Sorkness CA, Li JT, et al. Asthma Control Test: reliability, validity, and responsiveness in patients not previously followed by asthma specialists. J Allergy Clin Immunol. 2006; 117:549-556.

24. Banoub RG, Hoehle LP, Phillips KM, et al. Depressed mood modulates impact of chronic rhinosinusitis symptoms on quality of life. J Allergy Clin Immunol Pract 2018.

25. Ivanova JI, Bergman R, Birnbaum HG, Colice GL, Silverman RA, McLaurin K. Effect of asthma exacerbations on health care costs among asthmatic patients with moderate and severe persistent asthma. Allergy Clin Immunol. 2012; 129:1229-1235.

26. 26. Campbell AP, Phillips KM, Hoehle LPet al. Association between Asthma and Chronic Rhinosinusitis Severity in the Context of Asthma Control. Otolaryngol Head Neck Surg 2018; 158:386-390.

27. Phillips KM, Bergmark RW, Hoehle LP, Caradonna DS, Gray ST, Sedaghat AR. Chronic rhinosinusitis exacerbations are differentially associated with lost productivity based on asthma status. Rhinology 2018; 56:323-329.

28. ten Brinke A, Sterk PJ, Masclee AAet al. Risk factors of frequent exacerbations in difficult-to-treat asthma. Eur Resp J. 2005; 26:812-818.

29. Brandao HV, Cruz CS, Pinheiro MC, et al. Risk factors for ER visits due to asthma exacerbations in patients enrolled in a program for the control of asthma and allergie rhinitis in Feira de Santana, Brazil. J Bras Pneumol. 2009; 35:1168-1173.

30. Schlosser RJ, Smith TL, Mace J, Soler ZM. Asthma quality of life and control after sinus surgery in patients with chronic rhinosinusitis. Allergy 2017; 72:483-491. 
31. Batra PS, Kern RC, Tripathi Aet al. Outcome analysis of endoscopic sinus surgery in patients with nasal polyps and asthma. Laryngoscope 2003; 113:1703-1706.

32. Sedaghat AR, Hoehle LP, Gray ST. Chronic rhinosinusitis control from the patient and physician perspectives. Laryngoscope Investig Otolaryngol 2018; 3:419-433.

33. Speth MM, Gaudin RA, Hoehle LPet al. Reciprocal Predictive Accuracy of Sinonasal Symptom Severity, Nasal Endoscopy, and Frequency of Past Chronic Rhinosinusitis Exacerbations. Otolaryngol Head Neck Surg 2018: 159:766-773.

34. Lin DC, Chandra RK, Tan BKet al. Association between severity of asthma and degree of chronic rhinosinusitis. Am J Rhinol Allergy 2011; 25:205-208
Ahmad R. Sedaghat, MD, PhD, FACS

Department of Otolaryngology -

Head and Neck Surgery

University of Cincinnati College of Medicine

Medical Sciences Building Room 6410

231 Albert Sabin Way

Cincinnati, $\mathrm{OH} 45267-0528$

USA

Tel: +001 513-558-4152

Fax: +001 513-558-3231

E-mail: ahmad.sedaghat@uc.edu

\section{ADVERTISEMENT}
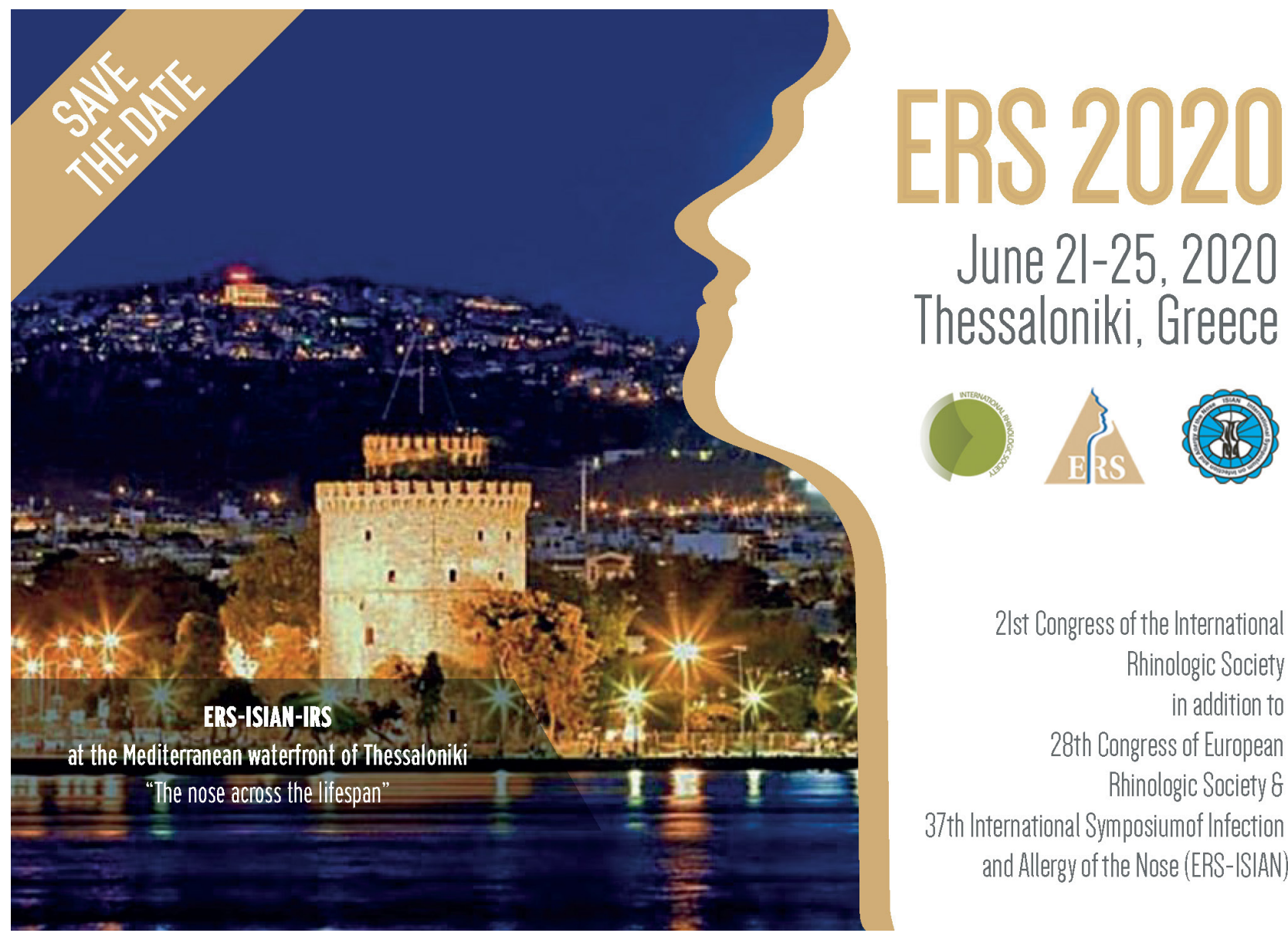

2|st Congress of the International

Rhinologic Society

in addition to

28th Congress of European

Rhinologic Society 8

37th International Symposiumof Infection and Allergy of the Nose (ERS-ISIAN) 